\title{
Surface modification effects on defect-related photoluminescence in colloidal CdS quantum dots
}

\section{TaeGi Lee, Kunio Shimura, DaeGwi Kim}

\begin{tabular}{|c|l|}
\hline Citation & Physical Chemistry Chemical Physics, 20(17); 11954-11958 \\
\hline Issue Date & 2018-05-07 \\
\hline Type & Journal Article \\
\hline Textversion & Author \\
\hline $\begin{array}{c}\text { Supplementary } \\
\text { files }\end{array}$ & Supplementary information is available at https://doi.org/10.1039/C7CP07812A. \\
\hline Relation & $\begin{array}{l}\text { The following article has been accepted by [Physical Chemistry Chemical Physics]. } \\
\text { After it is published, it will be found at https://doi.org/10.1039/C7CP07812A }\end{array}$ \\
\hline DOI & \begin{tabular}{l}
$10.1039 / \mathrm{C} 7 \mathrm{CP} 07812 \mathrm{~A}$ \\
\hline
\end{tabular} \\
\hline
\end{tabular}

\author{
Self-Archiving by Author(s) \\ Placed on: Osaka City University
}

LEE T., SHIMURA K., \& KIM D. (2018). Surface modification effects on defect-related photoluminescence in colloidal CdS quantum dots. Physical Chemistry Chemical Physics. 20, 11954-11958. 
Surface modification effects on defect-related photoluminescence in colloidal CdS quantum dots

TaeGi Lee, Kunio Shimura, and DaeGwi Kim*

Department of Applied Physics, Graduate School of Engineering, Osaka City University, 3-3-138, Sugimoto, Sumiyoshi-ku, Osaka 558-8585, Japan

*E-mail: tegi@a-phys.eng.osaka-cu.ac.jp

We investigated the effects of surface modification on the defect-related photoluminescence (PL) band in colloidal CdS quantum dots (QDs). A size-selective photoetching process and a surface modification technique with a $\mathrm{Cd}(\mathrm{OH})_{2}$ layer enabled the preparation of size-controlled CdS QDs with high PL efficiency. The Stokes shift of the defect-related PL band before and after the surface modification was $\sim 1.0$ $\mathrm{eV}$ and $\sim 0.63 \mathrm{eV}$, respectively. This difference in the Stokes shifts suggests that the origin of the defect-related PL band was changed by the surface modification. Analysis by X-ray photoelectron spectroscopy revealed that the surface of the CdS QDs before and after the surface modification was $\mathrm{S}$ rich and $\mathrm{Cd}$ rich, respectively. These results suggest that Cd-vacancy acceptors and S-vacancy donors affect PL processes in CdS QDs before and after the surface modification, respectively. 


\section{INTRODUCTION}

In semiconductor quantum dots (QDs), the quantum nature of carriers and/or excitons becomes apparent because of the QDs' finite size [1,2]. Over the past three decades, semiconductor QDs have attracted considerable attention by researchers who have endeavored to elucidate the size dependence of physical and/or chemical properties of QDs [3-6]. The absorption and photoluminescence (PL) properties of QDs are highly size dependent; the preparation of QDs with uniform size is therefore important.

Surface structures of semiconductor QDs strongly affect the relaxation process of photogenerated carriers and/or excitons because the surface-to-volume ratios of QDs are much larger than those of bulk crystals. Thus, control of the surface structure of QDs is essential for controlling their PL characteristics. Preparing core/shell QDs is a typical technique for improving QDs' PL properties. In this approach, core QDs are covered with a shell layer to improve the QDs' PL efficiency; the wide-bandgap semiconductor $\mathrm{ZnS}$ is the most commonly used shell material [7-9]. The CdSe/ZnS core/shell QDs have been a model material for QD studies $[10,11]$.

Most previous studies on the PL properties of colloidal QDs have focused on their band-edge PL; thus, little attention has been devoted to defect-related PL in QDs. Controlling the defect states and understanding the details of defect-related PL is considered important for producing high-efficiency luminescent materials.

In the present work, we investigate the origin of defect-related PL in colloidal CdS QDs. The QDs of CdS were prepared by a colloidal method. In the PL spectrum before the surface modification, the defect-related PL band with a large Stokes shift of $\sim 1 \mathrm{eV}$ is dominant, and the band-edge PL band is negligibly weak. The surface modification with a $\mathrm{Cd}(\mathrm{OH})_{2}$ layer strongly activates the band-edge PL band and is accompanied by a 
decrease of the Stokes shift of a defect-related PL band to $\sim 0.6 \mathrm{eV}$. The difference in the Stokes shift suggests that the origin of the defect-related PL band is changed by the surface modification. The effect of the surface modification on defect-related PL is also discussed on the basis of the results of X-ray photoelectron spectroscopy (XPS) measurements.

\section{EXPERIMENTS}

CdS QDs were synthesized by injecting $\mathrm{H}_{2} \mathrm{~S}$ gas $(0.2 \mathrm{mmol})$ into $100 \mathrm{ml}$ of an aqueous solution containing $0.2 \quad \mathrm{mmol} \quad \mathrm{Cd}\left(\mathrm{ClO}_{4}\right)_{2}$ and $0.2 \mathrm{mmol}$ sodium hexametaphosphate, a dispersion agent for colloids. The as-grown CdS QDs have a wide size distribution of 30\%-40\%. By size-selective photoetching, the size-distribution width can be narrowed to $5 \%$ or better $[12,13]$. The size-selective photoetching treatment was performed by irradiating the sample solution with a monochromatic light. For the photoetching of the QDs, a 500-W Xe lamp was used as a light source. Monochromatic light was obtained using interference filters; the full-width at half-maximum intensity of the resulting monochromatic light was $\sim 10 \mathrm{~nm}$. The surface modification is performed by the addition of $\mathrm{Cd}\left(\mathrm{ClO}_{4}\right)_{2}$ after adjusting $\mathrm{pH}$ of the solution to the alkaline region [12]. This process leads to the formation of a $\mathrm{Cd}(\mathrm{OH})_{2}$ layer on the surface of the CdS QDs [13, 14].

For XPS measurements, the monolayer structure of CdS QDs was prepared as follows. The Si substrates were cleaned by immersion in fresh piranha solution (1/3 (v/v) mixture of $30 \% \mathrm{H}_{2} \mathrm{O}_{2}$ and $98 \% \mathrm{H}_{2} \mathrm{SO}_{4}$ ) for 20 min (caution: piranha solution reacts violently with organic materials). The substrates were then rinsed with water and used immediately after cleaning. In the beginning of the sample preparation, the adhesion layer of polyelectrolytes of positively charged poly(diallyldimethylammonium 
chloride) (PDDA) was deposited to enhance the binding of QDs. The monolayer structure of CdS QDs was then deposited onto the PDDA layer.

Absorption spectra were recorded using a spectrophotometer with a spectral resolution of $0.2 \mathrm{~nm}$. For PL measurements, the 325-nm line of a He-Cd laser was used as an excitation-light source. The spectral resolution was $0.2 \mathrm{~nm}$ in the PL measurements. For the measurements of PL-decay profiles for the longer time region than $\sim 10 \mathrm{~ns}$, third-harmonic-generation $(\mathrm{THG})$ light $(355 \mathrm{~nm})$ of a laser-diode pumped yttrium aluminum garnet (YAG) laser with a repetition of $10 \mathrm{kHz}$ was used as the excitation light. The PL-decay profiles were obtained by a time-correlated single-photon counting method. For the measurement for a few nanosecond time region, a mode-locked Ti: sapphire pulse laser (400 nm center wavelength, 150 fs pulse duration, $82 \mathrm{MHz}$ repetition rate) was used as the excitation light source. The decay curves were detected and analyzed with a streak camera with the time resolution of $\sim 50 \mathrm{ps}$.

\section{RESULTS AND DISCUSSION}

Figure 1(a) shows the absorption spectrum of the as-grown CdS QDs. The absorption structure is observed on the high-energy side of the bandgap energy of $\sim 2.5$ $\mathrm{eV}$ in the bulk crystal. According to the theory of the quantum confinement effect in QDs [15], the lowest exciton energy in spherical QDs of II-VI semiconductors is usually explained well by the electron-hole individual confinement model, where the radius of QDs is smaller than the exciton Bohr radius. In this case, the lowest exciton energy in a spherical QD with radius $R$ is given by [15]

$$
E=E_{g}+\frac{\hbar^{2}}{2 \mu}\left(\frac{\pi}{R}\right)^{2}-1.8 \frac{e^{2}}{\varepsilon R}
$$

where $E_{g}, \mu$, and $\varepsilon$ are the bandgap energy, reduced mass, and dielectric constant, 
respectively. On the basis of eq. (1), the mean radius of the CdS QDs was estimated to be $2.1 \mathrm{~nm}$ from the absorption energy when the parameters of $m_{\mathrm{e}}=0.19, m_{\mathrm{h}}=0.70$, and $E_{\mathrm{g}}=2.50 \mathrm{eV}$ were used $[16,17]$

The spectral width of the absorption spectrum, however, is very broad, and no peak structure is observed because of the wide size distribution of the QDs. The problem of an inhomogeneous QD size can be solved via size-selective photoetching $[13,18]$. The monochromatic light obtained using interference filters was irradiated onto the sample solution, and the photon energy of the irradiation light was increased in stages until a clear absorption peak was observed [12, 13].

Figure 1(b) shows the absorption spectrum of the CdS QDs after the photoetching process. The observation of an absorption peak indicates that the size distribution of the CdS QDs is considerably narrow. The observed absorption spectrum is a superposition of absorption spectra of individual QDs with size distribution. The line-shape analysis with use of a combination of Gaussian line shapes of the excitonic absorption is a reasonable and convenient method for estimating the magnitude of the size-distribution width [19]. Open circles in Figure S1 shows the calculated results. The average radius and size-distribution width estimated from the line-shape analysis are $2.1 \mathrm{~nm}$ and $5 \%$, respectively. Figure S2 shows TEM images of CdS QDs before and after the photoetching. It is obvious that the size distribution before the photoetching is large and is reduced by the photoetching.

Figure 2 shows the absorption and PL spectra of the CdS QDs before and after the surface modification process. Upon the surface modification with a $\mathrm{Cd}(\mathrm{OH})_{2}$ layer $[13$, 14], the absorption peak shifted to the low-energy side. The effective confinement size is well known to be increased by the formation of a shell, and this increase in effective confinement size weakens the quantum confinement effect in core/shell QDs. Thus, the 
low-energy shift of the absorption energy after the dipping treatment is due to $\mathrm{Cd}(\mathrm{OH})_{2}$ shell formation on the CdS QDs.

In the PL spectrum before the surface modification (Fig. 2(a)), the defect-related PL band with a large Stokes shift of $\sim 1 \mathrm{eV}$ is dominant and the band-edge PL band is negligibly weak. The PL spectrum of the surface-modified QDs is clearly drastically changed (Fig. 2(b)). The band-edge PL band is strongly activated by the surface modification and is observed as the main PL band. Spanhel et al. proposed the following mechanism for the surface modification [14]. At high $\mathrm{pH}$ levels, $\mathrm{SH}^{-}$groups of the surface of CdS QDs are converted into $\mathrm{S}^{2-}$ ions, which then bind excess $\mathrm{Cd}^{2+}$ ions, possibly in the form of $\mathrm{S}^{2-}-\mathrm{Cd}^{2+}-\mathrm{OH}^{-}$structures. The surface modification resulting from the removal of $\mathrm{SH}^{-}$groups and the accumulation of $\mathrm{Cd}^{2+}$ ions on the surface might substantially diminish the nonradiative process, thereby drastically increasing the band-edge PL intensity [14].

Figure 3 (a) shows PL-decay profile of band-edge PL before the surface modification in CdS QDs with an average radius of $2.1 \mathrm{~nm}$. The dotted curve shows the time characteristic of the excitation laser light, and the response time of the measurement system can be estimated to be $\sim 50$ ps. The band-edge PL before the surface modification exhibits a very fast decay comparable to that of the excitation light. Fig. 3 (b) shows PL-decay profile of band-edge PL after the surface modification. It is obvious that the PL-decay profile changes significantly compared with that before the surface modification. The PL-decay profile after the modification shows a slow decay in microsecond range.

Since the splitting energy between the bright-exciton and dark-exciton state in $\mathrm{CdS}$ QDs with a radius of $2.1 \mathrm{~nm}$ is larger than the thermal energy at room temperature [13], the dark exciton state greatly contributes to PL processes even at room temperature. In 
the CdS QDs before the surface modification, the emission intensity is weak and a very fast attenuation profile is observed because the influence of the non-radiative recombination process is very large. On the other hand, the non-radiative recombination on the QD surface is greatly suppressed by the surface modification, and as a result, the PL intensity is increased and the PL-decay profile is changed drastically.

To investigate the surface-modification-induced changes in the surface composition of the QDs, XPS measurements were performed. Figure 4(a) and 4(b) show Cd 3d and S 2p XPS spectra, respectively, of CdS QDs before the surface modification. The signals observed at $405.1 \mathrm{eV}$ and $411.8 \mathrm{eV}$ correspond to those of $\mathrm{Cd} 3 \mathrm{~d}_{5 / 2}$ and $\mathrm{Cd} 3 \mathrm{~d}_{3 / 2}$, respectively [20, 21]. In addition, the signals observed at $161.4 \mathrm{eV}$ and $167.5 \mathrm{eV}$ correspond to those of sulfur in $\mathrm{CdS}$ and oxidized sulfur in $\mathrm{CdSO}_{4}[20,21]$. The results of XPS measurements for the $3 d$ orbital of $\mathrm{Cd}$ and the $2 \mathrm{p}$ orbital of $\mathrm{S}$ after the surface modification are shown in Fig. 5(a) and 5(b), respectively. It is obvious that the signal related to the $3 \mathrm{~d}$ orbital of $\mathrm{Cd}$ is strongly enhanced. The peak intensity ratios of $\mathrm{Cd} 3 \mathrm{~d}_{5 / 2}$ at $405.1 \mathrm{eV}$ to $\mathrm{S} 2 \mathrm{p}$ at $161.4 \mathrm{eV}$ before and after the surface modification are 17 and 400 , respectively. Similar XPS spectra were obtained for CdS QDs with the different size. The dependence of the $\mathrm{Cd} / \mathrm{S}$ signal intensity ratio on the QD diameter before and after the surface modification were listed in the Table $\mathrm{S} 1$. In all samples, the $\mathrm{Cd} / \mathrm{S}$ signal intensity ratio is drastically increased by the surface modification. Thus, a Cd-rich surface layer is likely formed on the CdS QDs after the surface modification. We note that the present XPS analysis results verify the appropriateness of the proposal in ref. [14] that the surfaces of CdS QDs before and after surface modification are S rich and Cd rich, respectively.

As noted in the discussion of Fig. 2, the band-edge PL band is strongly activated by the surface modification. Furthermore, the peak energy of the defect-related PL band is 
shifted to the higher-energy side by the surface modification. The Stokes shift of the defect-related PL band before and after the surface modification is $\sim 1 \mathrm{eV}$ and $\sim 0.6 \mathrm{eV}$, respectively. This difference in the Stokes shift suggests that the origin of the defect-related PL band is changed by the surface modification.

To discuss the change of the Stokes shift due to the surface modification, we prepared several samples with different QD sizes and measured their absorption and PL spectra before and after surface modification. Figure 6 shows the dependence of the Stokes shift of the defect-related PL band on the mean diameter of the CdS QDs. Open circles and rectangles represent the Stokes shift of the defect-related PL band before and after the surface modification, respectively. The broken lines denote the average value of the Stokes-shift. The results indicate that the Stokes shift of the defect-related PL band before and after the surface modification is $\sim 1.0 \mathrm{eV}$ and $\sim 0.63 \mathrm{eV}$, respectively.

The aforementioned difference in the Stokes shift suggests that the origin of the defect-related PL band is changed by the surface modification. Elavarthi et al. reported that sulfur vacancy $\left(\mathrm{V}_{\mathrm{S}}\right)$ donors were located $0.59 \mathrm{eV}$ below the conduction band in $\mathrm{CdS}$ [22]. Furthermore, Susa et al. reported that the energy level of Cd-vacancy $\left(\mathrm{V}_{\mathrm{Cd}}\right)$ acceptors is located approximately $1.1 \mathrm{eV}$ above the valence band [23]. The values of $1.1 \mathrm{eV}$ and $0.59 \mathrm{eV}$ are similar to the magnitude of the Stokes shift of the defect-related PL band before and after the surface modification, respectively. Thus, $\mathrm{V}_{\mathrm{Cd}}\left(\mathrm{V}_{\mathrm{S}}\right)$ likely affects the PL processes in CdS QDs before (after) the surface modification. This interpretation is consistent with the experimental XPS analysis results that the surface of CdS QDs is S rich and Cd rich before and after the surface modification, respectively. On the basis of these results, for the origin of the defect-related PL band, we conclude that, before the surface modification, $\mathrm{V}_{\mathrm{Cd}}$ strongly affects PL processes and the defect-related PL band originates from the recombination between electrons and holes 
trapped at $\mathrm{V}_{\mathrm{Cd}}$ (Fig. 7(a)). However, after the surface modification resulting in a $\mathrm{Cd}(\mathrm{OH})_{2}$ layer, the band-edge PL is strongly activated and the Stokes shift of the defect-related PL band is reduced to $\sim 0.63 \mathrm{eV}$. The origin of the defect-related PL band after the surface modification is the recombination between electrons trapped at $\mathrm{V}_{\mathrm{S}}$ and holes, as schematically shown in Fig. 7(b).

\section{CONCLUSION}

The effects of surface modification on the defect-related band in the PL spectra of colloidal CdS QDs were investigated. The CdS QDs with a narrow size distribution were prepared using size-selective photoetching. Through surface modification with the $\mathrm{Cd}(\mathrm{OH})_{2}$ layer, the PL properties were dramatically improved. Furthermore, the magnitude of the Stokes shift of the defect-related PL band was dramatically changed by the surface modification, reflecting that the origin of the defect-related PL band is changed by the surface modification. The XPS analysis results clearly demonstrate that the surface of CdS QDs before and after the surface modification is S rich and Cd rich, respectively. On the basis of these results, the defect-related PL bands before and after the surface modification are considered to originate from the recombination of electrons and holes trapped at $\mathrm{V}_{\mathrm{Cd}}$ and from the recombination of electrons trapped at $\mathrm{V}_{\mathrm{S}}$ and holes, respectively.

\section{ACKNOWLEDGEMENTS}

This work was supported partially by a Grant-in-Aid for Scientific Research (B) (No. 17H03538) from the Japan Society for the Promotion of Science. 


\section{References}

[1] L. Brus, Appl. Phys. A, 1991, 53, 465.

[2] Y. Wang and N. Herron, J. Phys. Chem., 1991, 95, 525.

[3] A. P. Alivisatos, Science, 1996, 271, 933.

[4] D. J. Norris and M. G. Bawendi, Phys. Rev. B, 1996, 53, 16338.

[5] D. J. Norris, A. L. Efros, M. Rosen, and M. G. Bawendi, Phys. Rev. B, 1996, 53, 16347.

[6] X. michalet, F. F. Pinaud, L. A. Bentolila, J. M. Tsay, S. Doose, J. J. Li, G. Sundaresan, A. M. Wu, S. S. Gambhir, and S. Weiss, Science, 2005, 307, 538.

[7] B. O. Dabbousi, J. Rodriguez-Viejo, F. V. Mikulec, J. R. Heine, H. Mattoussi, R. Ober, K. F. Jensen, and M. G. Bawendi, J. Phys. Chem. B, 1997, 101, 9463.

[8] V. V. Nikesh, and S. Mahamuni, Semicond. Sci. Technol., 2001, 16, 687.

[9] L. Li, A. Pandey, D. J. Werder, B. P. Khanal, J. M. Pietryga, and V. I. Klimov, J. Am. Chem. Soc., 2011, 133, 1176.

[10] Y. Chen, T. Ji, and Z. Rosenzweig, Nano Lett., 2013, 3, 581.

[11] A. V. Baranov, Y. P. Rakovich, J. F. Donegan, T. S. Perova, R. A. Moore, D. V. Talapin, A. L. Rogach, Y. Masumoto, and I. Nabiev, Phys. Rev. B, 2003, 68, 165306.

[12] D. Kim, N. Teratani, K. Mizoguchi, H. Nishimura, and M. Nakayama, Int. J. Mod. Phys. B, 2001, 15, 3829.

[13] D. Kim, T. Mishima, K. Tomihira, and M. Nakayama, J. Phys. Chem. C, 2008, 112, 10668.

[14] L. Spanhel, M. Haase, H. Weller, and A. Henglein, J. Am. Chem. Soc., 1987, 109, 5649.

[15] L. E. Brus, J. Chem. Phys., 1984, 80, 4403. 
[16] D. Schooss, A. Mews, A. Eychmüller, H. Weller, Phys. Rev. B, 1994, 49, 17072.

[17] Q. Zeng, X. Kong, Y. Sun, Y. Zhang, L. Tu, J. Zhao and H. Zhang, J. Phys. Chem. $C, 2008, \mathbf{1 1 2}, 8587$.

[18] H. Matsumoto, T. Sakata, H. Mori, and H. Yoneyama, J. Phys. Chem., 1996, 100, 13781.

[19] D. Kim, N. Teratani, and M. Nakayama, Jpn. J. Appl. Phys., 2002, 41, 5064.

[20] A. V. Okotrub, I. P. Asanov, S. V. Larionov, A. G. Kudashov, T. G. Leonova, and L. G. Bulusheva, Phys. Chm. Chem. Phys. 2010, 12, 10871.

[21] A. Hamdi, D. P. Ferreira, A. M. Ferraria, D. S. Conceição, L. F. Vieira Ferreira, A. P. Carapeto, S. Boufi, S. Bouattour, and A.M. Botelho do Rego, J. Nanomaterials 2016, 2016, 6581691.

[22] P. Elavarthi, A. A. Kumar, G. Murali, and D. A. Reddy, J. Alloys Compd., 2016, 656, 510.

[23] N. Susa, H. Watanabe, and M. Wada, Jpn. J. Appl. Phys., 1976, 15, 2365. 


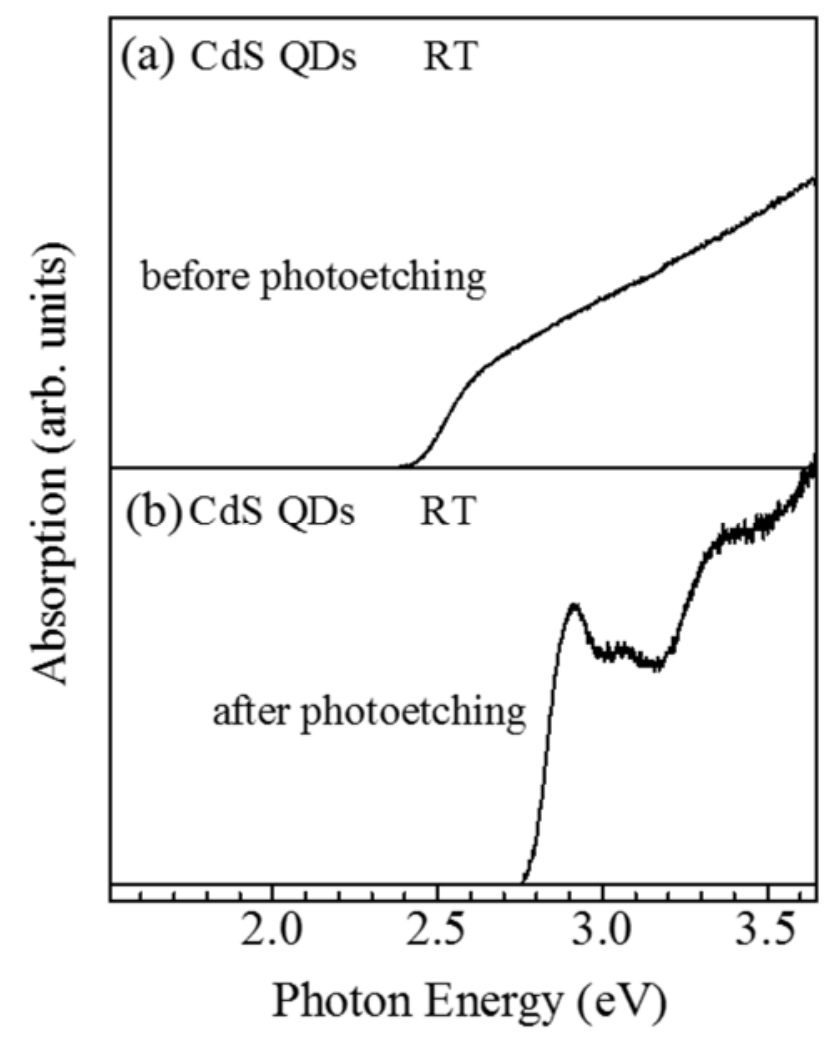

Fig. 1. (a) Absorption spectrum of as-grown CdS QDs. (b) Absorption spectrum of CdS QDs after the photoetching process. 


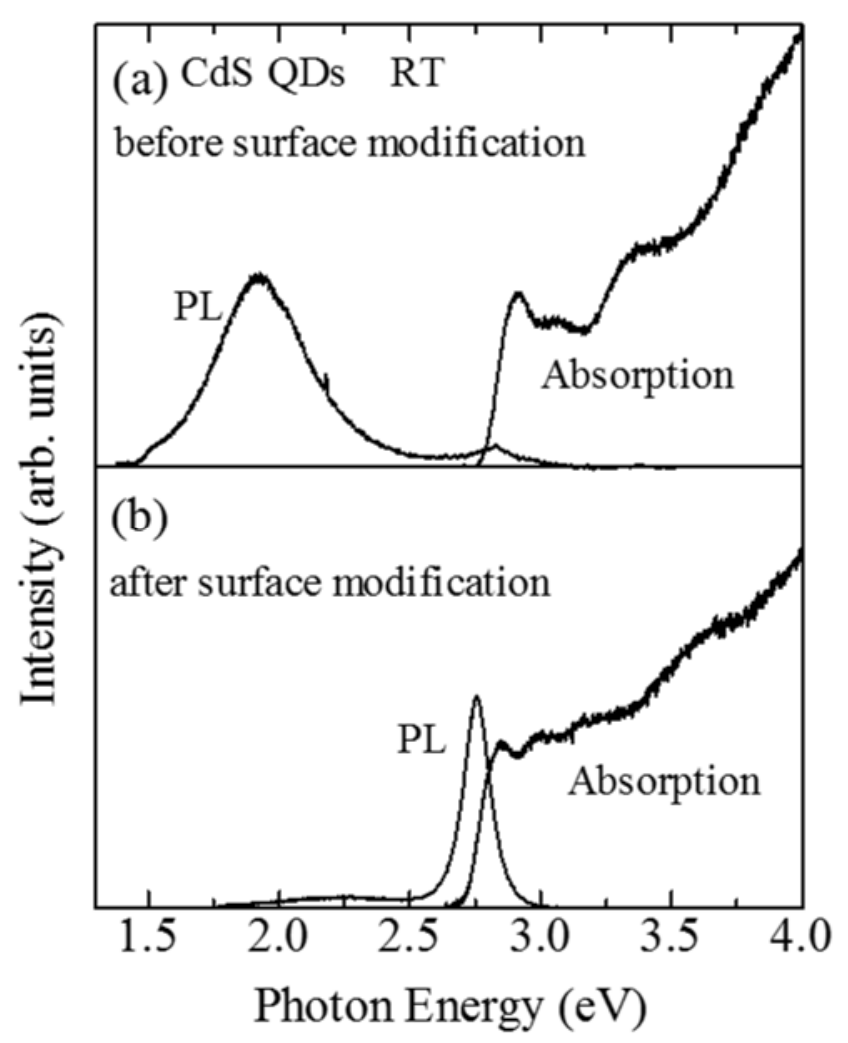

Fig. 2. Absorption and PL spectra of CdS QDs (a) before and (b) after the surface modification. 

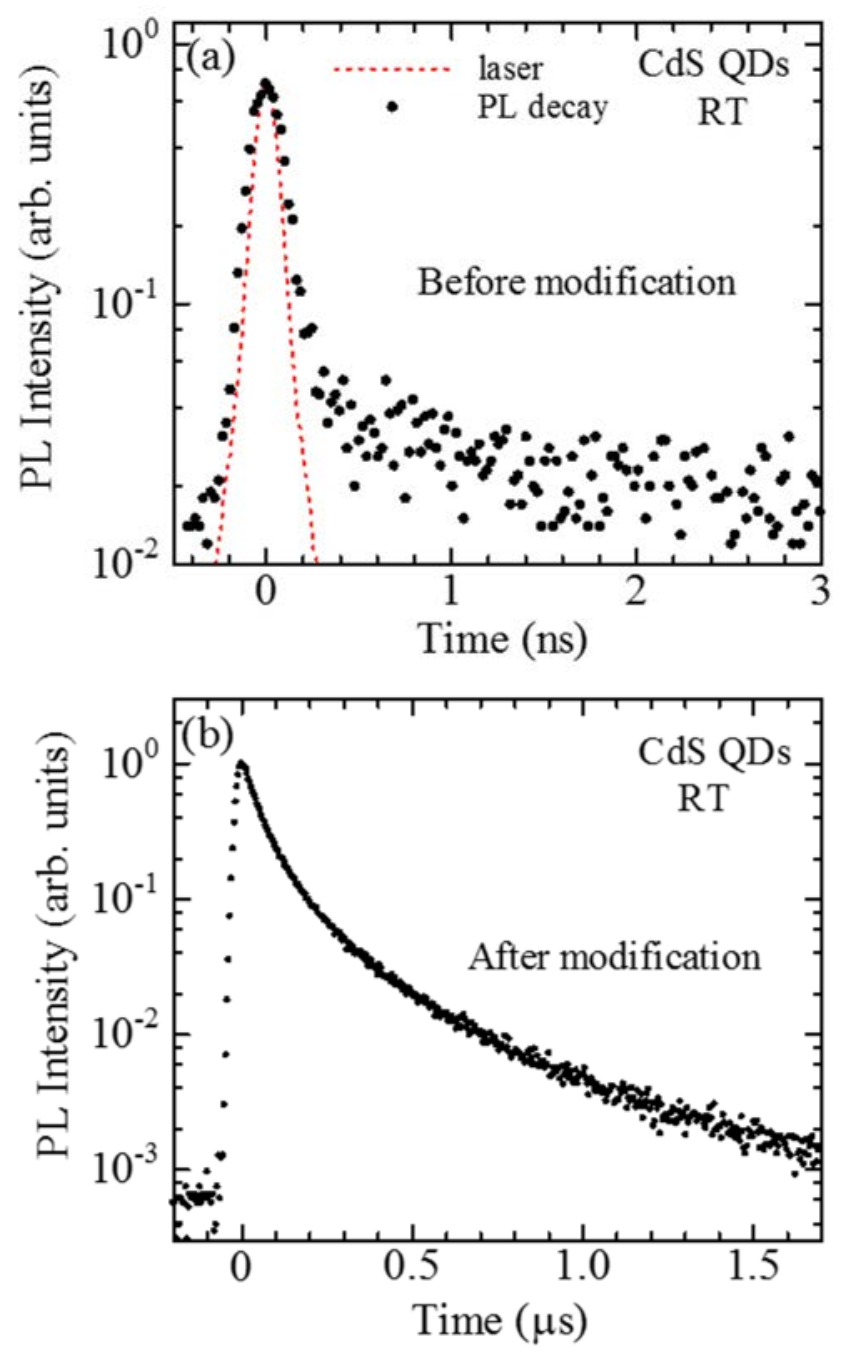

Fig. 3. (a) PL-decay profile of band-edge PL before the surface modification in CdS QDs with an average radius of $2.1 \mathrm{~nm}$. The dotted curve shows the time characteristic of the excitation laser light. (b) PL-decay profile of band-edge PL after the surface modification. 

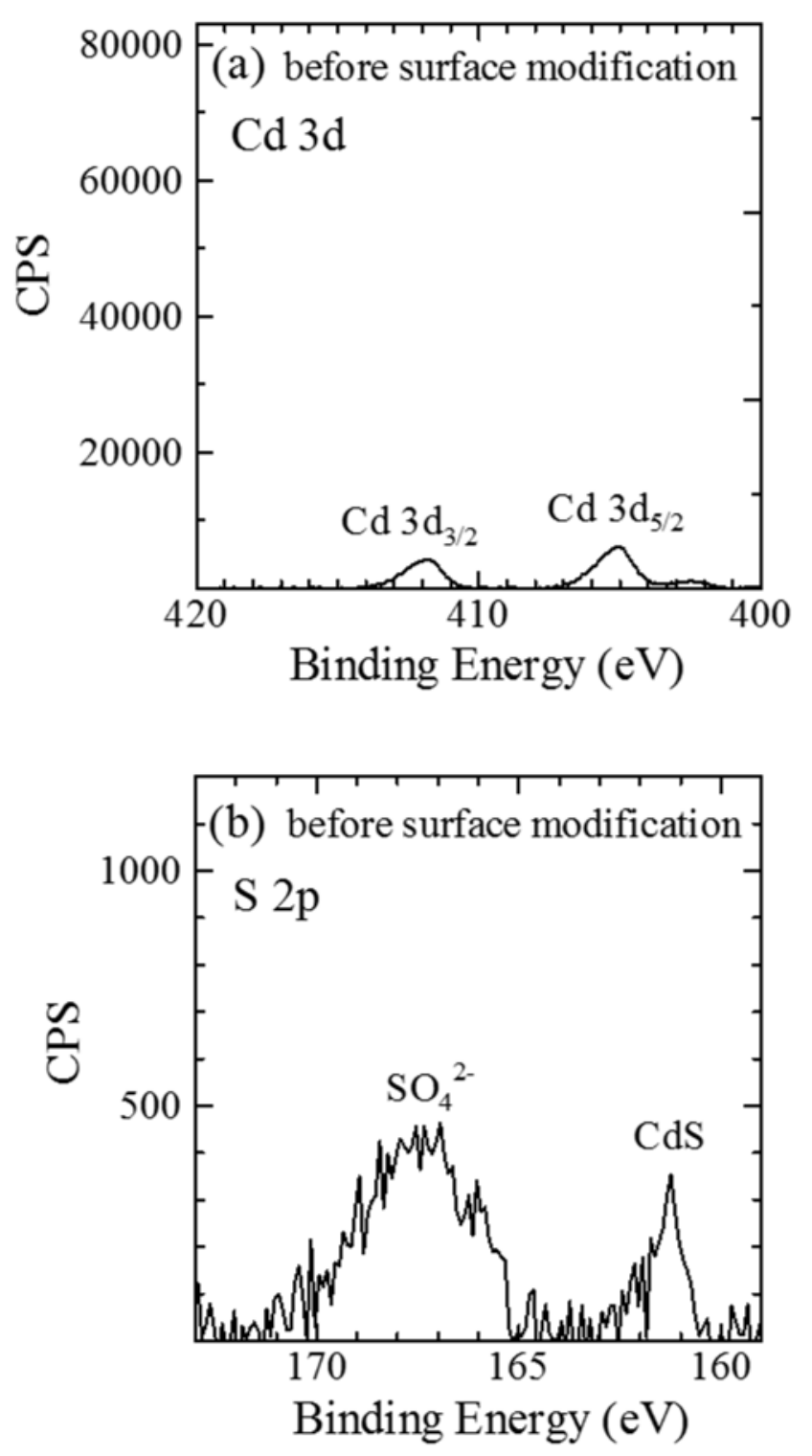

Fig. 4. Results of XPS measurements for (a) $3 d$ orbitals of Cd and (b) the $2 s$ orbitals of $\mathrm{S}$ before the surface modification. 

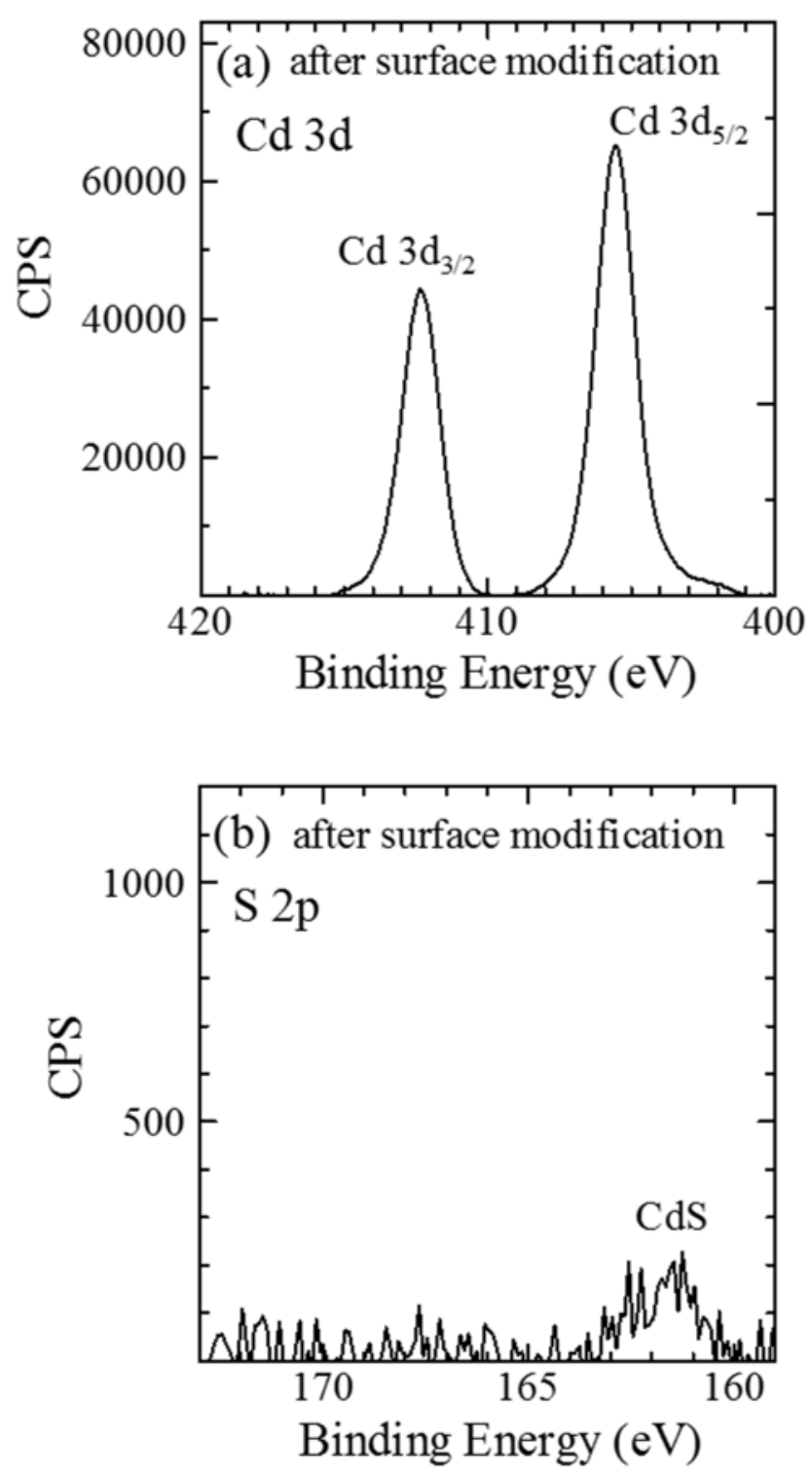

Fig. 5. Results of XPS measurements for (a) the $3 d$ orbital of Cd and (b) the $2 s$ orbital of $\mathrm{S}$ after the surface modification. 


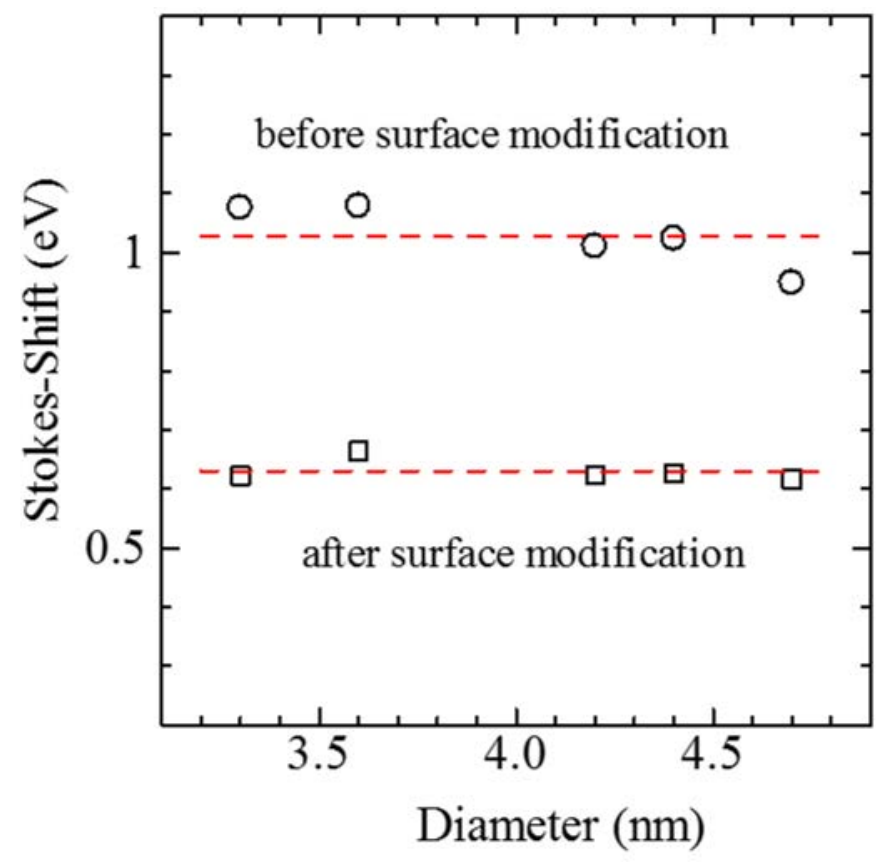

Fig. 6. The dependence of the Stokes shift of the defect-related PL band on the mean diameter of CdS QDs. Open circles and rectangles represent the Stokes shift of the defect-related PL band before and after the surface modification, respectively. The broken lines denote the average value of the Stokes shift. 
(a) Before surface modification

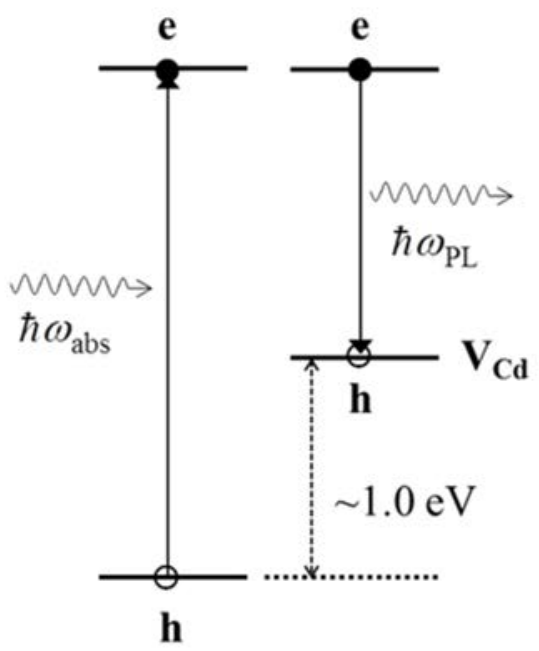

(b) After surface modification

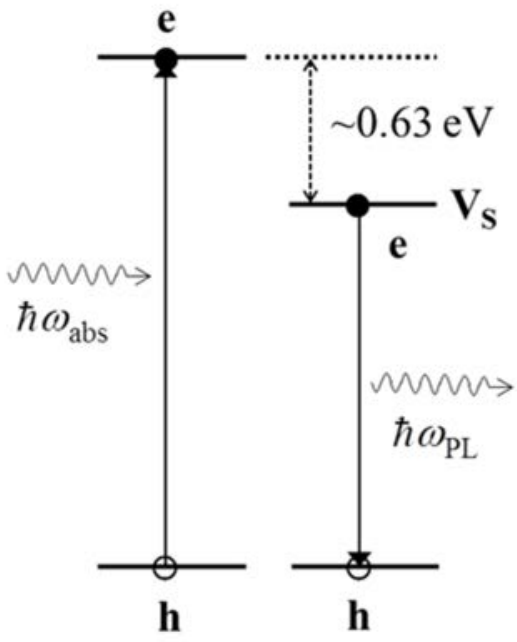

Fig. 7. Schematic energy level diagram corresponding to CdS QDs (a) before and (b) after the surface modification. $\mathrm{V}_{\mathrm{Cd}}$ and $\mathrm{V}_{\mathrm{S}}$ represent cadmium vacancy acceptors and sulfur vacancy donors, respectively. 


\section{Electronic supplementary information}

Surface modification effects on defect-related photoluminescence in colloidal CdS quantum dots

TaeGi Lee, Kunio Shimura, and DaeGwi Kim*

Department of Applied Physics, Graduate School of Engineering, Osaka City University, 3-3-138, Sugimoto, Sumiyoshi-ku, Osaka 558-8585, Japan

*E-mail: tegi@a-phys.eng.osaka-cu.ac.jp

\section{$\underline{\text { Table of Contents }}$}

1. Line-shape analysis of absorption spectra

2. TEM images of CdS QDs (a) before and (b) after the photoetching

3. Absorption and PL spectra of CdS QDs with different sizes before and after the surface modification

4. The dependence of XPS signals on the QD size before and after the surface modification 


\section{Line-shape analysis of absorption spectra}

The observed absorption spectrum $F_{a b s}(E)$ is considered to be a superposition of absorption spectra $A(R, E)$ of individual QDs with size distribution $N(R)$ and can be expressed as

$$
F_{a b s}(E)=\int A(R, E) N(R) d R .
$$

By substituting the absorption peak energy into eq. (1), the mean radius can be obtained.

Since the line width of $A(R, E)$ of each QDs is sufficiently narrow as compared with the width of the total spectrum $F_{a b s}(E)$, it does not affect the calculation of $F_{a b s}(E)$ given by eq. (1). Therefore, the size distribution width $\sigma$ remains as the only adjustable parameter in the calculation of $F_{a b s}(E)$. Open circles in Figure S1 shows the calculated results. The average radius and size-distribution width estimated from the line-shape analysis are $2.1 \mathrm{~nm}$ and $5 \%$, respectively.

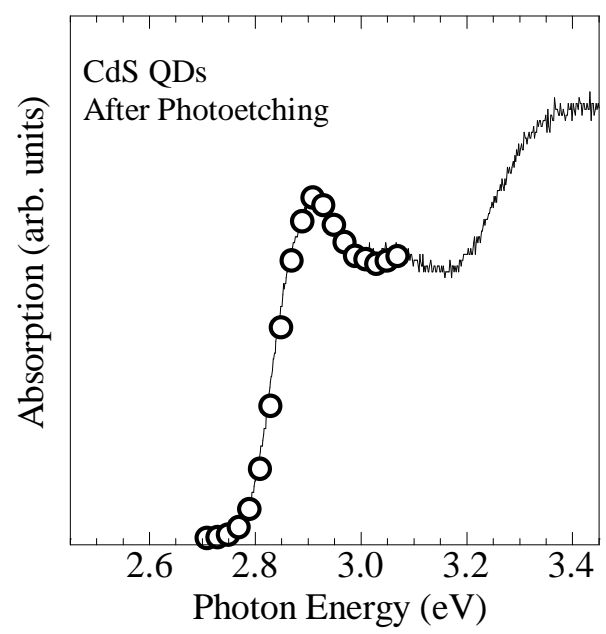

Fig. S1. Absorption spectrum of CdS QDs after the photoetching. The open circles show the results of the line-shape analysis. 


\section{TEM images of CdS QDs before and after the photoetching.}

Figure S2 shows TEM images of CdS QDs before and after the photoetching. It is obvious that the size distribution before the photoetching is large and is reduced by the photoetching.
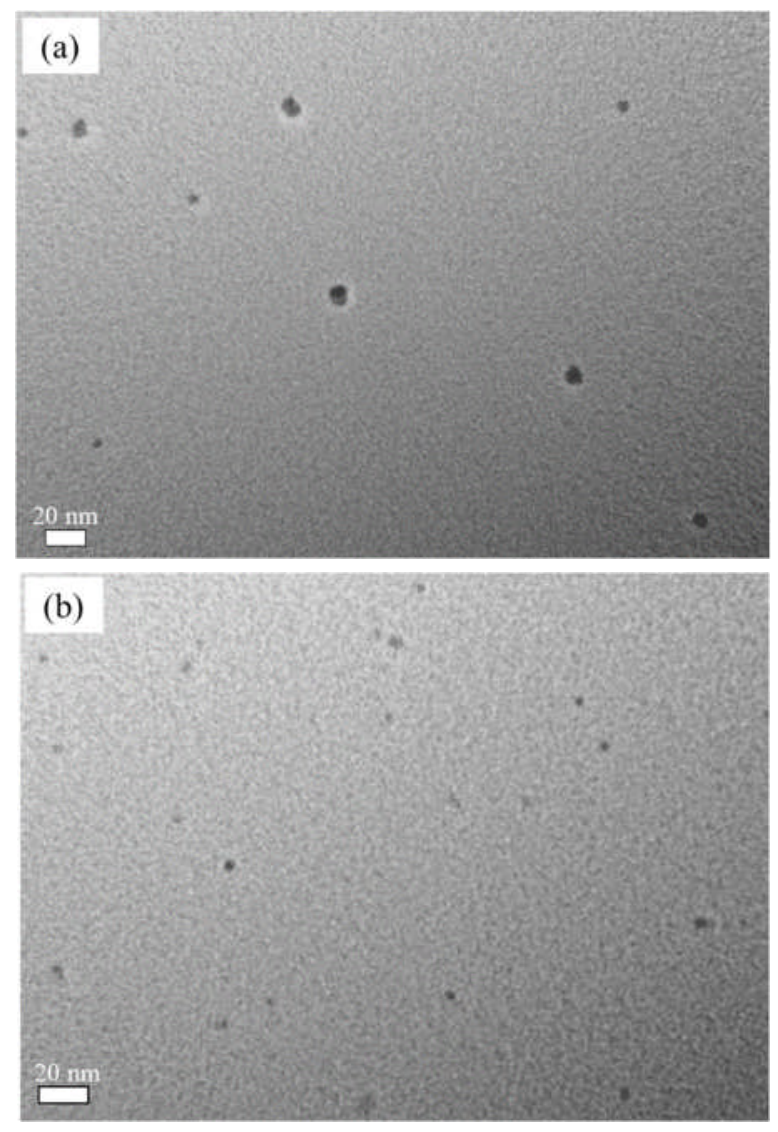

Fig. S2. TEM images of CdS QDs (a) before and (b) after the photoetching. 
3. Absorption and PL spectra of CdS QDs with different sizes before and after the surface modification

Figures S3 and S4 show absorption and PL spectra of CdS QDs with different sizes before and after the surface modification.

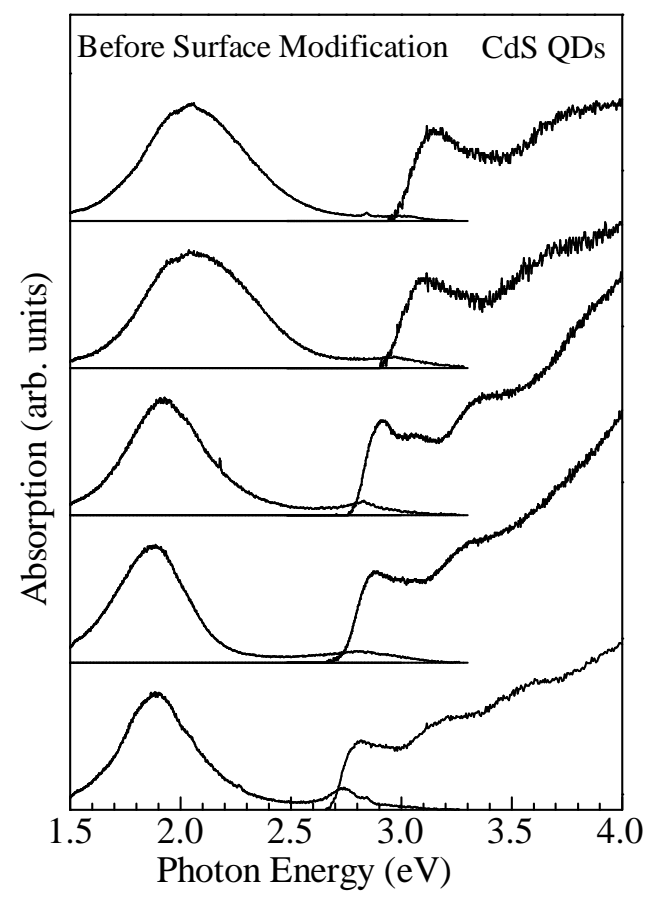

Fig. S3. Absorption and PL spectra of CdS QDs with different sizes before the surface modification. 


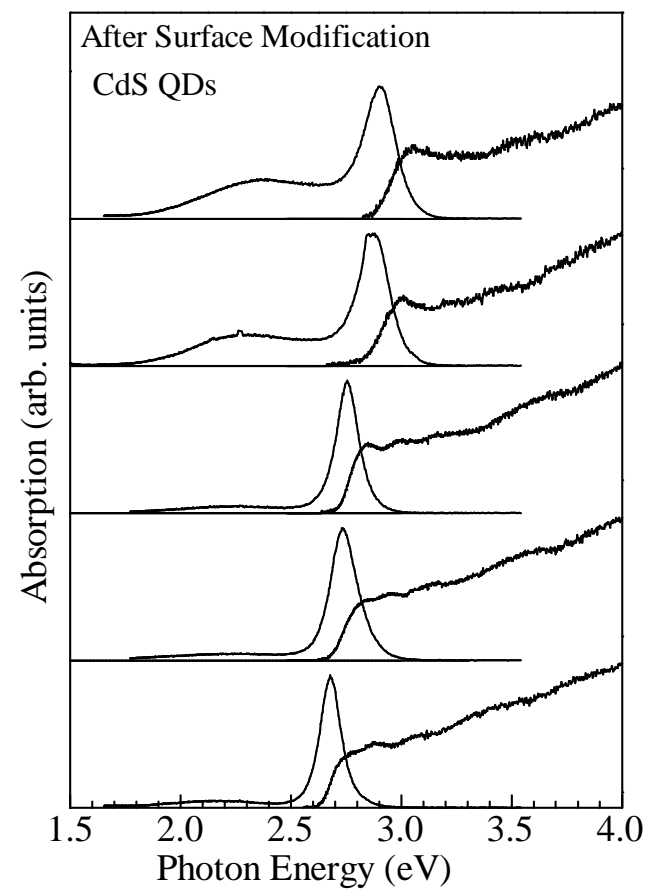

Fig. S4. Absorption and PL spectra of CdS QDs with different sizes after the surface modification. 
4. The dependence of XPS signals on the QD size before and after the surface modification

Similar XPS spectra were obtained for CdS QDs with the different size. The dependence of the $\mathrm{Cd} / \mathrm{S}$ signal intensity ratio on the QD size before and after the surface modification were listed in the Table S1.

Table S1. The XPS intensity ratio of $\mathrm{Cd} 3 \mathrm{~d}_{5 / 2}$ to $\mathrm{S} 2 \mathrm{p}$ before and after the surface modification for CdS QDs with the different diameter.

\begin{tabular}{|c|c|c|c|c|}
\hline QD diameter & $4.7 \mathrm{~nm}$ & $4.4 \mathrm{~nm}$ & $4.2 \mathrm{~nm}$ & $3.6 \mathrm{~nm}$ \\
\hline Before surface modification & 17 & 27 & 55 & 64 \\
\hline After surface modification & 400 & 310 & 450 & 1100 \\
\hline
\end{tabular}

